\title{
Misinformation and Misdiagnosis in Freeman-Burian syndrome
}

\author{
Mikaela I Poling ${ }^{1}$ and Craig R Dufresne ${ }^{1,2}$ \\ ${ }^{1}$ Craig R Dufresne, MD, PC \\ ${ }^{2}$ Virginia Commonwealth University
}

January 31, 2022

Correspondence: Mikaela I Poling, 8501 Arlington BLVD, Ste 420, Fairfax, VA 22031, USA; Tel. +1 304-460-9038; Fax +1 703-207-2002; E-mail: research@duplastics.com

Author Contributions: Both authors contributed equally.

Acknowledgments: We thank CM Poling, M Pocket, W Cloud, and ES Tu. This manuscript is dedicated to the memory of Calvin Yang, formerly of the 2nd Battalion, 27th Infantry Regiment "Wolfhounds" of the US Army, who lost his life to complications of posttraumatic stress disorder several years after serving.

Conflict of Interest: The authors have no financial or other competing interests to disclose.

Funding: This work was unfunded.

Word Count: 810 (Body)

\section{KEYWORDS}

Freeman-Burian syndrome; Freeman-Sheldon syndrome; malignant hyperthermia; craniocarpotarsal dystrophy; whistling face syndrome; distal arthrogryposis type 2A; case reports; rare diseases; craniofacial abnormalities

We read with interest the article, "Freeman-Sheldon Syndrome with Stiff Knee Gait - A Case Report," by Drs Sehrawat, Sural, Sugumar, Khan, Kar, and Jeyaraman.[1] It is wonderful to see this rare syndrome discussed. Unfortunately, there were several unclear or inaccurate points, and recent publications were omitted, suggesting an incomplete literature search. As Freeman-Sheldon syndrome, now Freeman-Burian syndrome (FBS),[2] is exquisitely rare, many who believe they have encountered it in clinical practice are eager to publish their experience, despite the perils.

The authors present FBS as a variant of distal arthrogryposis,[1] but FBS is a unique condition.[3] Though similar in physical appearance, FBS is genetically unique and has a unique clinical course.[3] Two studies have provided evidence that certain craniofacial findings are pathognomonic for FBS, [4-5] and a meta-analysis found distal arthrogryposes are not required for diagnosis.[4] Though many continue to do so, FBS can no longer be cogently classed as a distal arthrogryposis syndrome in the face of such evidence, and the most reasonable classification seems to be as a complex congenital myopathic craniofacial syndrome.[3]

The authors next discuss the patient's copper beaten skull appearance.[1] They do not detail any workup or treatment.[1] The authors postulate it is related to craniosynostosis but quickly dismiss it. It is concerning that they seemed to have focused on a suspected diagnosis of a well-known rare syndrome and ignored a concerning neurologic finding.

The authors describe FBS as an, "autosomal dominant trait and in rare cases as autosomal recessive and x-linked recessive".[1] It is believed that inherited cases either have a parent with clinical evidence of FBS or rarely are germline mosaicisms[4-6]. 
The authors next speak of, "skeletal abnormalities" and "skeletal malformations".[1] In the syndrome, problems involving the skeletal system are secondary effects of the primary myopathic process of fibrose tissue replacement of normal muscle fibers.[7] This fibrose tissue acts as constricting bands, the way collagen behaves in severe burns.[7] This is correlated with in vitro molecular myophysiology observations showing problems with the metabolic process for contraction and extreme muscle stiffness that reduces muscular work and power.[7] Misunderstanding of etiology in FBS has led to inappropriate treatment plans, especially surgeries, and has resulted in tragic, lifelong impairments.[4,7-8] We applaud the authors for their caution toward surgery and explanation of its potential for unfavorable outcomes.[1]

Unlike many similar case reports to which we have responded over the past 2-years, the authors clearly state the standard clinical diagnostic criteria (microstomia, pursed lips, deep nasolabial folds, and $\mathrm{H}$ or V-shaped chin defect and two major arthrogryposes-typically, camptodactyly with ulnar deviation and equinovarus).[1,4-5] They also provide a somewhat fair description of FBS, except as noted.[1] A major failing of their description of FBS is their summary of findings suggestive of FBS, Sheldon-Hall syndrome (SHS), and Schwartz Jampel syndrome.[1] The table lists some required findings as, "Features not favoring FSS".[1,4-5] Generally, the construction of the table is misleading, as structured diagnostic criteria exist for FBS and SHS.[4-5]

The authors also present a very clear description of their patient, with acceptable figures.[1] It is mystifying that-despite correctly stating the FBS diagnostic criteria twice-they diagnosed their patient with FBS, though she clearly did not meet the diagnostic criteria.[1,4-5] The patient lacked the $\mathrm{V}$ or $\mathrm{H}$-shaped chin defect and had a small mouth but not microstomia, as judged from included photographs.[1] The patient's appearance seems consistent with SHS, exhibiting a small mouth, short neck, small chin, obvious nasolabial folds, and triangular face.[1] Such an error is not uncommon; FBS has an estimated false-positive rate of $30-60 \% \cdot[4-5]$

The authors state there is an association of malignant hyperthermia (MH) with FBS and urge caution.[1] Some patients with FBS do, indeed, develop hyperpyrexia during general anesthesia, but these hyperpyrexia events, which may include tachycardia and increased muscle rigidity, respond to ibuprofen and also occur where a malignant hyperthermia protocol was followed and in stressful, non-operative stress situations.[9] There is no evidence that MH is associated with FBS, though FBS anesthesia practice guidelines still suggest following an $\mathrm{MH}$ protocol.[9]

Prenatal ultrasound is not considered diagnostic, and prenatal diagnosis is not considered generally feasible.[6] As suggested by the authors, molecular diagnosis is very expensive and is not clinically helpful or needed, given the strong correlation of the clinical diagnostic criteria with the presence of MYH3 mutations.[1,6] For women with clinically diagnosed FBS undergoing in vitro fertilization and wishing to avoid use of an FBS-affected egg, polar bodies testing has been used successfully, as the authors correctly state.[1,6]

Ten English-language case reports have been published between 2020-2022 purportedly describing FBS that contained similar, preventable errors.[1,10-18] Not conducting a thorough literature search and omitting recent articles was the common denominator among the articles.[19] In trying to address the shortcomings of each, we have responded to all ten, with four letters already published.[19-22] This article illustrates the potential perils of describing a rare condition despite the best intentions.

\section{References}

1. Sehrawat S, Sural S, Sugumar PA, Khan S, Kar S, Jeyaraman M. Freeman-Sheldon Syndrome with Stiff Knee Gait - A Case Report. Journal of Orthopaedic Case Reports 2021 November;11(11):64-68. doi: $10.13107 /$ jocr.2021.v11.i11.2518

2. Poling MI, Dufresne CR. Revisiting the many names of Freeman-Sheldon syndrome. J Craniofac Surg. 2018;29(8):2176-2178. doi: 10.1097/SCS.0000000000004802

3. Poling MI, Dufresne CR. Head first, not feet first: Freeman-Sheldon syndrome as primarily a craniofacial condition. Cleft Palate-Craniofac J. 2018;55(5):787-788. doi: 10.1177/1055665617753482 
4. Poling MI, Dufresne CR, Chamberlain RL. Findings, Phenotypes, Diagnostic Accuracy, and Treatment in Freeman-Burian Syndrome. J Craniofac Surg. 2020;31:1063-1069. doi: 10.1097/SCS.0000000000006299

5. Stevenson DA, Carey JC, Palumbos J, Rutherford A, Dolcourt J, Bamshad MJ. Clinical characteristics and natural history of Freeman-Sheldon syndrome. Pediatrics. 2006;117:754-762. doi: 10.1542 /peds. $2005-1219$

6. Hague J, Delon I, Brugger K, Martin H, Abbs S, Park SM. Molecularly proven mosaicism in phenotypically normal parent of a girl with Freeman-Sheldon Syndrome caused by a pathogenic MYH3 mutation. Am J Med Genet A. 2016;170(6):1608-1612. doi: 10.1002/ajmg.a.37631

7. Poling MI, Dufresne CR, Chamberlain RL. Freeman-Burian syndrome. Orphanet J Rare Dis. 2019;14:14. doi: 10.1186/s13023-018-0984-2

8. Poling MI, Dufresne CR, McCormick RJ. Identification and Recent Approaches for Evaluation and Management of Rehabilitation Concerns for Patients with Freeman-Burian Syndrome: Principles for Global Treatment. J Ped Genet. 2020;09(03):158-163. doi: 10.1055/s-0040-1710339

9. Poling MI, Dufresne CR. Freeman-Burian syndrome. Anästh Intensivmed. 2019;60(1):S8-S17. doi: 10.19224/ai2019.S008

10. Kamal G, Shah SB, Gupta A. Anesthesia Challenges in the Management of Freeman-Sheldon Syndrome: Report of Two Cases and Literature Review. AANA J. 2020;88(1):35-38. PMID: 32008616.

11. Park J, Kang SW, Choi WA, Lee Y, Cho HE. Precise Pulmonary Function Evaluation and Management of a Patient With Freeman-Sheldon Syndrome Associated With Recurrent Pneumonia and Chronic Respiratory Insufficiency. Ann Rehabil Med. 2020;44(2):165-170. doi: 10.5535/arm.2020.44.2.165

12. Wróblewska-Seniuk K, Jarząbek-Bielecka G, Kędzia W. Freeman-Sheldon syndrome - a course of the disease from birth to adulthood. Clin Exp Obstet Gynecol. 2020;47(6): 978-982. doi: $10.31083 /$ j.ceog.2020.06.5430

13. Sato Boku A, Sento Y, Hasegawa T, Tsutsumi K, Kamimura Y, So M, Kako E, Sobue K. Anesthetic management of a patient with Freeman-Sheldon syndrome undergoing oral surgery: a case report. Clin Case Rep. 2021;9(6):e04358. Published 2021 Jun 9. doi: 10.1002/ccr3.4358

14. Kiliçbay F, Küçük Kurtulgan H, Sidar Duman Y, Tunç G. A Case Report of Freeman-Sheldon Syndrome with Gastrointestinal Dysmotility in a Premature Newborn Delivered Due to Polyhydramnios. Turkiye Klinikleri J Case Rep. 2021;29(4):230-3. doi: 10.5336/caserep.2021-83838.

15. Wu TM, Chin LS. Treatment results of teratologic arthrogryposis in a case of Freeman-Sheldon syndrome: A 25-year follow-up. Formos J Surg. 2021;54:66-9. doi: 10.4103/fjs.fjs_79_20

16. Sreeja KS, Sahoo J, Barman A, Baral D. Holistic rehabilitation of a rare case of distal arthrogryposis: Freeman Sheldon syndrome. International Journal of Scientific Research. 2021;10(5). Available at: https: //www. worldwidejournals. com/international-journal-of-scientific-research(IJSR) /fileview/holistic-rehabilitation-of-a-rare-case-of-distal-arthrogryposisfreeman-sheldon-syndrome_May_2021_6121064717_ 8726505.pdf

17. Fayed M, Giska MA, Shievitz RC, Attali A, Younger J. Emergent Cesarean Delivery in a Patient With Freeman-Sheldon Syndrome Complicated by Preeclampsia, Acute Pulmonary Embolism, and Pulmonary Edema: A Case Report. Cureus. 2021;13(12):e20802. doi: 10.7759/cureus.20802

18. Bowman S, Noble G, Rahmani B, Mets M, Ranaivo HR, Castelluccio V. A case of blepharophimosis: Freeman Sheldon syndrome. Ophthalmic Genet. 2021. doi: 10.1080/13816810.2021.1989603

19. Poling MI, Dufresne CR. Unsafe Care and Misunderstanding Diagnosis in Freeman-Burian syndrome: Problems in Writing Case Reports Involving Rare Conditions and Strategies for Improvement. Version: 3. Authorea [Preprint]. January 26, 2022. doi: 10.22541/au.164303994.43963430/v3

20. Poling MI, Dufresne CR. Letter. AANA J. 2020;88(5):54. Available at: https://www . aana.com/docs/ default-source/aana-journal-web-documents-1/20-oct-letters.pdf?sfvrsn=7aa229e4_6

21. Poling MI, Dufresne CR. Letter: Precise Pulmonary Function Evaluation and Management of a Patient With Freeman-Sheldon Syndrome Associated With Recurrent Pneumonia and Chronic Respiratory Insufficiency. Ann Rehabil Med. 2020;44(5):409-410. doi: 10.5535/arm.20110

22. Poling MI, Dufresne CR. Accuracy of Facts About Freeman-Sheldon syndrome. Clin Exp Obstet Gyne- 
col. 2021;48(5):997-998. doi: 10.31083/j.ceog4805160 